Canadian Journal of Civil Engineering

Canadian

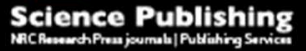
Revue canadienne de génie civil

\title{
Breaking into emergency shuttle service: Aspects and impacts of retracting buses from existing scheduled bus services
}

\begin{tabular}{|r|l|}
\hline Journal: & Canadian Journal of Civil Engineering \\
\hline Manuscript ID & cjce-2017-0294.R2 \\
\hline Manuscript Type: & Article \\
\hline Date Submitted by the Author: & 02-Jan-2018 \\
\hline $\begin{array}{r}\text { Complete List of Authors: } \\
\text { Is the invited manuscript for } \\
\text { consideration in a Special } \\
\text { Issue? : }\end{array}$ & $\begin{array}{l}\text { Diab, Ehab; University of Toronto, Department of Civil Engineering } \\
\text { Shalaby, Amer; University of Toronto, }\end{array}$ \\
\hline Keyword: & subway, system interruption, streetcars, shuttle service, bus bridging \\
\hline \multicolumn{2}{|c|}{} \\
\hline
\end{tabular}




\section{retracting buses from existing scheduled bus services}

* Ehab Diab, Ph.D.

University of Toronto

Department of Civil Engineering (Transportation Engineering)

35 St. George Street, Toronto, Ontario M5S 1A4 Canada, Room: SF3001M

Email: ehab.diab@utoronto.ca

\section{Guangnan Feng}

University of Toronto, Engineering science

35 St. George Street, Toronto, Ontario M5S 1A4 Canada,

Email: nansen.feng@mail.utoronto.ca

Amer Shalaby, Ph.D., P.Eng.

University of Toronto

Department of Civil Engineering (Transportation Engineering)

35 St. George Street, Toronto, Ontario M5S 1A4 Canada, Room: SF3001H

Email: amer@ecf.utoronto.ca 


\section{ABSTRACT}

28 High-quality transit service is a vital aspect of any modern city. When unexpected interruptions

29 to the transit service occur, they reduce the quality of service provided to the public. One of the 30 main strategies that is employed to deal with rail service interruptions is "bus bridging," whereby

31 buses from scheduled services are deployed to offer shuttle services. Very few efforts are found

32 in the literature that investigate this policy effectiveness. Therefore, this study aims at exploring

33 the different aspects and impacts of retracting buses from scheduled services in response to

34 subway and streetcar service interruptions in Toronto. It explores the size of the deployment, as

35 well as the system response and recovery times using detailed subway and streetcar shuttle

36 service reports collected in 2015. The paper shows remarkable fluctuations not only in the

37 utilized number of shuttle service buses over time, but also on the service response and recovery

38 times.

39 Key words: subway, streetcars, system interruption, public transit, shuttle service, bus 40 bridging

41 


\section{1. INTRODUCTION}

45 Public transit is considered an essential service for any city, due to its indispensable role in

46 supporting the daily activities of city residents. When unexpected interruptions to the public

47 transit service occur, they reduce the quality of service provided to the public and diminish the

48 system's ability to retain existing customers and attract new ones. As discussed in the literature,

49 transit users are usually concerned not only about their average travel and waiting times, but also

50 about the uncertainty and variability in transit service, which affects their decision-making and

51 time-planning processes (Bates et al. 2001; Nam et al. 2005; Noland and Polak 2002). Thus,

52 transit agencies and authorities implement several disruption management strategies (or

53 emergency response plans) to mitigate and reduce the impact of unexpected disruptions and

54 incidents on users' costs. Several studies have been conducted around these issues, focusing

55 mainly on proposing conceptual frameworks, testing different approaches for disruption

56 management or categorizing incidents according to some criteria (Codina and Marin 2010;

57 Kepaptsoglou and Karlaftis 2009; Schmöcker et al. 2005; Wang et al. 2014). To name just a few

58 examples, Pender et al. (2015) tested a new method to explore the economic viability of

59 providing dedicated bus service reserved for bus bridging purposes for rail system disruption.

60 Bus bridging refers to the strategy of providing temporary bus shuttle services that restore

61 connectivity between disrupted subway or railway stations (Kepaptsoglou and Karlaftis 2009).

62 Darmanin et al. (2010) developed a mathematical model to minimize commuter discomfort when

63 a service disruption occurs in the Metro system in Melbourne. Another recent study by van der

64 Hurk et al. (2016) proposed a mathematical model to estimate shuttle line frequencies under

65 budget constraints for the Massachusetts Bay Transportation Authority, Boston, Massachusetts. 
Notwithstanding the previous efforts concerning the provision of a dedicated bus fleet

67 reserved for bus bridging purposes, according to a recent international survey of 71 transit agencies regarding disruption recovery strategies, about $45 \%$ of the responding transit agencies reported retracting buses from existing scheduled bus service to deal with rail transit service disruptions. This has been done with no reserved buses for bus-bridging purposes. Toronto

71 Transit Commission (TTC), the public transit provider in the City of Toronto, Canada, is one of

72 these transit agencies (Pender et al. 2013). Interestingly, the TTC noted in this survey that by

73 doing that "you may in fact be simply shifting the problem or causing additional ones. " In view

74 of that, and due to the fact that there has been little effort in the literature to document and

75 explore the aspects and impacts of retracting buses from scheduled bus services, this research

76 aims at filling this gap. In fact, this disruption recovery strategy presents a challenge to transit

77 agencies, since sourcing of buses can be problematic at some locations and at some time periods.

78 In addition, the effects of retracting buses from existing scheduled must be considered and

79 integrated by transit planners during various operational stages to add the appropriate amount of

80 recovery time, if possible, or to redirect bus system users to other routes that suffer no reduction

81 in their frequencies.

Other researchers focused on exploring other important operational, managerial and user

83 behavioural aspects of rail service disruptions. For example, recent efforts developed and

84 executed a transit user behaviour survey and modelled user's travel behaviour in response to 85 subway service interruptions in Toronto (Lin 2017; Lin et al. 2017). A second study focused on understanding the impact of subway service interruptions on the service performance of both bus

87 and streetcar routes that are within a short walking distance from affected subway stations (Diab 88 and Shalaby 2017). Another recent study explored the managerial framework used by different 
transit agencies to deal with unplanned rail service disruptions (Pender et al. 2013). However, these and similar studies have not tackled the various operational aspects of retracting buses from scheduled services to offer emergency shuttle services nor analyzed this strategy using actual operational data collected from a real-world system. Therefore, the main aim of the presented study is to explore the different aspects and impacts of retracting buses from scheduled services in response to subway and streetcar service interruptions in the City of Toronto. The paper explores the size and impacts of emergency shuttle service deployment, as well as the system response and recovery times using detailed subway and streetcar shuttle service reports collected in 2015 by the TTC.

\section{DATA AND RESEARCH METHOD}

This study focuses on the City of Toronto, which is the largest city in Canada and the fourth largest in North America, with a total of 2.8 million inhabitants in 2015. The city's population is expected to increase considerably by $32 \%$ to reach 3.7 million in 2041 (Ontario Ministry of Finance 2015), adding more pressure on the current public transport system. The TTC operates a multimodal transit system consisting of four subway lines, 11 streetcars lines, and 141 bus routes (TTC 2013), serving more than 2.7 million passengers on a daily basis (American Public Transportation Association (APTA) 2013). The TTC subway network extends to a total length of $68 \mathrm{~km}$ serving 69 stations, while the streetcar route network extends to a total of $104 \mathrm{~km}$ serving 685 stops. Around 1.3 million passengers-trips per day used the subway system in 2013, while about 300,000 passenger-trips per day were made using the streetcar system (TTC 2012). The TTC bus system is comprised of seven different divisions, where each bus route is operated and monitored by a specific division based on its geographic location. All bus routes are coloured in Figure 1 according to the division they are managed by. 
The data used for this study includes the shuttle bus service reports acquired from the

113 TTC's Route Management Department for year 2015. A shuttle bus service log (or report) is

114 generated when an incident in either the streetcar or subway systems occurs and the TTC deploys

115 emergency shuttle service. Each incident is documented in one report which includes detailed

116 information on both the incident and deployed shuttle bus service, specifically the incident type,

117 date, time started, time cleared and location, as well as the number of shuttle buses requested and 118 assigned from different divisions. The report also includes detailed information on each assigned

119 shuttle bus including the vehicle number, original route number, assigned run number, time off

120 route to serve in the shuttle service, arrival time to the shuttle route and returning time to its

121 original route. These reports are filled manually (i.e., handwritten) in many cases by both the

122 Route Management Department (the Control Centre) and bus division(s), and they are normally

123 stored by the Control Centre for one calendar year before disposal. In total, more than 6000

124 pages, belonging to a total of 1094 shuttle service reports, were scanned, digitized and used in 125 this study. This unique dataset represents a rich resource to better understand the aspects and 126 impacts of retracting buses from scheduled service at the system level. The following analysis is

127 based on the available data from all shuttle service reports; however, missing information can be 128 found occasionally. To ensure accurate results, only data entries with complete information for a 129 specific analysis were used. For instance, a record with no information on the time a given bus 130 was retracted from its original route was used only in the geographical and incident analyses but 131 not used in the response time analysis (discussed later). 
This research employs various measures to explore the challenges associated with service resumption and recovery following subway and streetcar disruptions. It explores the magnitude of the problem by investigating the number of incidents, average delay per incident as well as the number of requested and assigned buses per incident. It also investigates the affected scheduled bus service by examining the number of buses retracted from each regular bus route. The system response time to service interruptions and system recovery time to return to normal operations are also explored. Finally, the paper explores the most frequent types of subway and streetcar incidents that impacted the scheduled bus service in 2015 . To better understand the system response time, this study breaks it into three major components as illustrated in Figure 2. The first component is the Initial Response Time, which is the time it takes the TTC's Route Management Department (Control Centre) from the incidents' start time to react to incidents. This portion of time includes the incident reporting time to the Control Centre and the time it takes the Control Centre to call bus divisions placing a request for shuttle service. Indeed, transit agency internal communication efficiency and effectiveness is a crucial issue in responding successfully to service interruptions, which was highlighted by several transit agencies (Pender et al. 2013). The second component is the Bus Pull out Time, which is the time it takes the TTC's bus divisions to unload and take buses off the scheduled service. If the Control Centre made more than one call, each bus pull out time is calculated according to the associated call. The third component is Bus Deadhead time, which is the travel time of buses from their original routes to the location of shuttle service.

In this study, we also classify buses into three categories to better recognise the challenge of providing shuttle bus service. These categories include buses requested by the Control Centre, buses assigned by divisions, and buses that actually arrived to the shuttle service location and 
157 provided the required emergency service. The main reason for having these different types of

158 buses is that not all requested buses by the Control Centre are normally provided by the bus

159 divisions. Also, some incidents could be cleared before the assigned buses arrive at the shuttle

160 service location. Thus, a Division response rate measure is developed to account for the number

161 of buses assigned by divisions divided by the total number of buses requested by the Control

162 Centre. Bus recovery time is the time it takes the buses to return to their scheduled service which

163 includes the Bus Service Time on the shuttle service and Bus Returning Time to original routes. A

164 conceptual workflow cycle for a shuttle bus service implementation is shown in Figure 2.

[FIGURE 2: TO BE ADDED HERE]

Figure 2: Regular workflow cycle for a shuttle bus service.

\section{3. TTC'S PROTOCOLS AND CURRENT PRACTICE}

168 The TTC employs specific protocols to initiate the emergency shuttle bus service. These

169 protocols mainly exist to deal with subway service interruptions. The decisions on shuttle service

170 deployment and number of assigned shuttle buses are based on the location of service

171 interruption along the subway system (central, east end, west end), subway line (main subway

172 lines, Scarborough Line, and Sheppard Line), day of week (weekday, weekends), time period

173 (AM, Midday, PM, etc.), number of subway affected stations (1-4 stations, 5-9 stations, 10+

174 stations) and the expected duration of subway interruption (1 to 30 minutes, $30+$ minutes). Table

1751 presents one example from these protocols. It shows the percentage and number of required

176 buses to deal with subway system service interruptions along the main subway lines (i.e.,

177 (Yonge-University-Spadina Line and Bloor-Danforth Line). The protocol categorizes the 
178 incidents by the expected duration of delay and number of affected (or closed) subway stations, 179 and it provides the required percentage of shuttle buses for each category according to the time 180 period of the day. For example, up to $10 \%$ of the buses serving on scheduled bus routes could be 181 retracted for deployment as shuttle buses, when a subway interruption is expected to last more 182 than 30 minutes and affects more than 10 subway stations. This percentage of buses is retracted 183 equally from all bus divisions, with no spatial consideration of the location of incident. As 184 different divisions have different numbers of buses in regular service, the number of buses to be 185 retracted from different divisions may vary so as to minimize the impact on divisions with small 186 fleets. Generally, similar protocols can be found requiring all divisions to source buses for 187 service interruptions along the main subway lines, except for a few exceptions, irrespective of 188 the incident location. This may lead to low efficiency in some cases where shuttle buses have to 189 travel a long distance if provided by a division which is not adjacent to the incident location.

Bus divisions are advised to retract buses from high frequency routes first. Trippers, 191 which stand for the extra buses scheduled for peak hour service, are always the first candidates. 192 However, other factors may affect the decision making such as bus driver's schedule and route 193 ridership. Out of courtesy, supervisors normally call the driver of the following bus to advise 194 them of pulling the bus ahead out of service to expect more than normal riders. In some unusual 195 cases, shuttle buses can be taken directly from garages, if spare drivers are available to operate 196 these buses. The TTC's Transit Control center calls bus division once the incident started to 197 place a request, however, in exceptional cases, it can call back the bus divisions up to three times 198 in total to request more buses or to follow up. The TTC has no strict geographical boundaries for 199 "central", "east end" and "west end" locations and it is left to the Transit Control's supervisors to 200 determine which shuttle guidelines to follow. Therefore, for the purpose of our study we divide 
201 the subway system into four different sections based on location to better understand the spatial

202 impacts of the shuttle service (Figure 1). In contrast to the subway system shuttle service

203 protocols, the TTC has no well-defined protocols for the streetcar shuttle service, and the

204 decisions for deploying such a service are made usually on an ad-hoc basis.

[TABLE 1: TO BE ADDED HERE]

Table 1: Number of required buses to be retracted for the shuttle service.

\section{OVERVIEW OF SUBWAY AND STREETCAR INCIDENTS}

\section{Number of Incidents and System Delay}

In 2015, the TTC dispatched shuttle bus services in response to 924 and 144 incidents in the streetcar and subway networks, respectively. Averaging at about 2.5/0.4 incidents per day in the streetcar/subway system, these incidents caused a total daily delay of 216.7 and 34.4 minutes in the streetcar and subway systems, respectively (see Figure 3-A). Here, delay refers to the incident duration, which was calculated based on the incident clearance time minus the start time, as indicated by shuttle service reports. Nevertheless, a simple division suggests that the average delay per incident is 86.7 minute for streetcars and 86.0 minutes for subway, which are very close. These lengthy delays are expected, since the analyzed data come from the shuttle service reports which mainly deal with 'Major' incidents that triggered shuttle service deployment.

As expected, the TTC experienced more incidents per day on weekdays than weekends for the streetcar and subway systems (Figure 3-B). These incidents are not equally distributed over the different day periods for the subway system (Figure 3-C). More incidents occurred 
222 during the mid-day and evening periods, for both the subway and streetcars systems.

223 Nevertheless, more delaying incidents occurred during the evening period for the streetcar 224 system than the subway system. The south (or Central) section which lies within the downtown 225 area had the lowest number of daily incidents (0.02 incidents per day) while the west section had 226 the highest number of incidents ( 0.14 incidents per day). This indicates more major incidents 227 occurring at the west section that required TTC to deploy the shuttle service. Also, this reflects 228 the TTC's efforts in clearing incidents more swiftly along the south section. It should be noted 229 that some subway incidents were reported at the entire route or a portion of route, instead of at a 230 stop level. Therefore, these incidents were removed from the spatial analysis because it was not 231 possible to link them to a specific location along the subway lines. Figure 3-E illustrates a clear 232 trend of more incidents and lengthier delays occurring during the winter season for both the 233 streetcar and subway systems.

[FIGURE 3: TO BE ADDED HERE]

Figure 3: A- Daily incidents and total daily delay by mode, B- Daily subway incidents by weekday vs. weekend, C- Daily incidents and average delay by time period, D- Daily subway incidents by location, and E- Daily incidents and total daily delay by month.

\section{The Number of Requested and Assigned Buses}

239 About 6500 buses were requested by the Control Centre to provide shuttle services in 2015, with an average of 23.1 and 3.5 buses per subway and streetcar incident, respectively (Figure 4-A).

241 This is intuitive due to the more frequent subway services and higher capacity of subway trains

242 (one subway train can carry up to1100 passengers while one streetcar may only carry between $243 \quad 100-200$ passengers). About $71 \%$ of the requested buses are assigned by bus divisions with a 
244

245

246

247

248

249

250

251

252

253

254

255

256

257

258

259

260

261

262

263

264

265

total of 17.1 and 2.3 buses per subway and streetcar incident, respectively. The breakdown of the number of requested and assigned buses by day of week and time of day (Figures 4-B and 4-C) depict similar patterns to those shown in the corresponding figures of the previous section.

Nevertheless, the number of requested and assigned buses differ slightly according to the time of the day. A slightly higher division response rate during peak hours can be observed (Figure 3-C), with a low response rate during the evening time. This perhaps reflects the higher availability of trippers during the peak periods which could diverted for shuttle service.

Regarding the geographic location of the subway incidents (Figure 4-D), the results for west and north sections agree with previous section too, showing that more buses are requested and assigned due to incidents. The south section requires few buses compared to other sections. This is related to the TTC's used protocols of deploying less number of buses at this central section due the availability of parallel regular streetcar service.

[FIGURE 4: TO BE ADDED HERE]

Figure 4: A- Buses requested and assigned per incident by mode, B- Daily buses requested and assigned by weekday vs. weekend, C- Daily buses requested and assigned by time period, D- Daily buses requested and assigned by location (for the subway system), E- Daily buses requested and assigned by month, and F- Daily shuttle bus service analysis for February 2015.

Figure 4-E gives the year's profile of the number of buses requested and assigned per month, which agree with number of incidents in Figure 3-E. The response rate, however, does not have a clear trend, and February shows an overall low response rate, despite having the greatest number of incidents and requested buses. Therefore, a new figure was generated to explore the daily trends during this month (Figure 4-F). As seen in the figure, an inconsistent response rate can be 
266 observed over the days of the month. This may highlight the need for more consistent policies

267 for bus assignment.

To summarize, several temporal, monthly and spatial trends in the number of incidents and their total delays can be observed across the subway and streetcar systems. The TTC's

270 protocols have been used to source the requested number of buses but monthly variations have

271 been observed, suggesting a thorough review of applying the used protocols may be in order.

272 There may be a need for more flexible protocols that enhance the system capacity of sourcing

273 buses during the winter season while relaxing these protocols during the other less demanding 274 seasons, such as summer.

\section{5. IMPACTED BUS ROUTES}

276 In 2015, an average of 1.25 buses per route and incident were retracted from 82 buses routes,

277 with a standard deviation of 0.3 buses. These routes represented a total of $65 \%$ of TTC's bus

278 routes in regular service. The analysis of 4,568 shuttle buses used in 900 incidents is presented in

279 this section. Figure 5-A shows that not all buses retracted from regular routes were fully

280 deployed to the target shuttle service location, due to some incidents getting cleared before the

281 shuttle bus arrival. Around $88 \%(5.2 / 5.9)$ and $85 \%(5.8 / 6.8)$ of the assigned buses were actually

282 utilized as a shuttle service for the streetcar and subway system, respectively. Some buses are

283 deployed from remote routes which may explain the less than perfect utilization rate. In addition,

284 the higher percentage of utilization for the streetcar system reflects its shorter response time

285 (discussed in the following section).

286 As shown in Figure 5-B, a percentage of buses were dispatched from garages as opposed 287 to scheduled route services. However, this percentage varies by the time period of day, with the 288 smallest parentage of buses dispatched from garages during the morning peak hour (14\%) and 
289 the evening period (15\%), while the largest during the midday period $(22 \%)$ and the afternoon 290 peak (23\%). This is expected and highlights the problem of sourcing buses during some periods

291 of the day due to the availability of spare drivers, which has been discussed in the literature

292 (Pender et al. 2013). In order to get a better idea about the impacts of retracting buses from 293 regular bus routes, the following discussion focuses on the top 20 bus routes from which shuttle 294 buses were sourced most frequently. From these 20 routes, around 2,000 shuttle buses were 295 extracted in 2015 (Figure 5-C), ranging from 53 to 209 buses per route. The figure also shows 296 the daily ridership per route in thousands, which is a reflection of the route offered capacity and 297 headway. The routes daily ridership ranges from 6,400 to 45,700 riders per day. The figure 298 shows that the number of assigned buses per route is not always proportionate to its ridership 299 level. On average, about 1.5 buses were retracted from each bus route during incident days 300 (Figure 5-D), despite differences in ridership levels. This practice may have considerable impacts 301 on the users of low frequency bus routes. The increase in users' waiting and travel times along 302 the routes would likely have negative impacts on users' perceptions and loyalty (Diab et al. 2017). In order to better understand the impacts of retracting buses from scheduled route service 304 on the performance and users of those routes, a separate study is recommended.

Figure 5: A- Daily assigned and in-shuttle buses by mode, B- Daily buses retracted from scheduled service and garages by time period, C- Top 20 bus routes that supplied buses for shuttle service in 


\section{RESPONSE TIME}

311 The response time is analyzed by mode, weekday vs. weekend, time period, month and location

312 for a total of 3,097 shuttle buses that covered 688 incidents. As seen in Figure 6-A, the total

313 response time to subway incidents is longer than the response time to streetcar incidents for all

314 three time components, with an average total time of 41.3 and 28.9 minutes per bus for the 315 subway and streetcar systems, respectively. These values are considerably shorter than the 316 average response time reported for rail transit bus-bridging of 90 minutes for a case study in

317 Australia (Pender et al. 2015). The difference between the subway and streetcar shuttle bus 318 response time can be attributed to the fewer shuttle buses required per streetcar incident, the 319 sources of which could be decided upon quickly (shorter initial response and pull out times) and 320 deployed from nearby bus routes (shorter deadhead times) relative to subway incidents which are

321 more complex and large-scale. The response time per bus on weekends is longer than weekdays

322 (Figure 6-B). This may be due to the limited staff resources and reduced bus fleet in service on 323 weekends which could limit shuttle bus options and delay the overall decision making process.

Figure 6-C indicates that the shuttle bus service is delivered more rapidly during peak

325 hours, with an average response time of 29 minutes per bus, compared to an average of 38

326 minutes per bus during off-peak periods. This may be due to the availability of trippers in most

327 routes during peak periods, offering rich and wide access to shuttle bus options. The shorter

328 deadhead times in peak periods, shown in the figure, supports this proposition. As expected, 329 longer response time can be observed during the winter months and into April (Figure 6-D) 330 mainly due to increases in pull out and deadhead times, reflecting the negative impacts of 331 weather conditions on bus service operations (Diab and El-Geneidy 2013). In contrast, the initial 332 response time does not vary much by season. 
Figure 6-E breaks down spatially the response time according to the incident location

along the subway system and bus division. This figure was constructed using a total of 79

subway incidents that caused the closure of 5 or more subway stations and required all bus divisions to provide a similar percentage of buses (see Table 1). As seen in the figure, some buses from some bus divisions can take an enormous amount of time to provide such a shuttle service. For instance, for incidents in the west section of the subway, the average response time of buses deployed from routes belonging to the Malvern division (which a northern-eastern bus division) was 58 minutes while the average delay for the subway system was around 87 minutes. There is a large probability that an incident in the west section could be cleared before shuttle buses arrive from the Malvern division. Longer response times inevitably increase the total waiting time for users that are stuck and frustrated while waiting for shuttle service, and they also increase the overall "clearance" time of incidents. As indicated previously, the southern section is a special case, representing the downtown core of the city of Toronto. For this section, the assignment of shuttle buses from all the Northern divisions (e.g., Malvern, Arrow, Wilson, Queensway) in response to subway incidents takes a considerable amount of time. This reflects a challenge of deploying buses from these locations in response to central subway service interruptions.

\section{[FIGURE 6: TO BE ADDED HERE]}

Figure 6: A- Average bus response time by mode, B- Average bus response time by weekday vs. weekend, C- Average bus response time by time period, D- Average bus response time by months, and E. Average bus response time by location in subway network and division. 


\section{RECOVERY TIME FOR REGULAR BUS ROUTES}

Recovery time, a key element of the overall shuttle service process, should be examined carefully by transit agencies, since clearing incidents swiftly will mean quicker return of shuttle buses to their original routes. Recovery time includes two components, namely service time on the shuttle service and returning time to the scheduled service after the incident is cleared. A total of 1,930 shuttle bus runs for 567 incidents were analyzed based on that. Interestingly, Figure 7-A and B show that the returning time was relatively longer than the response time discussed in the previous section, with an average of 44.4 and 32.4 minutes per bus for the subway and streetcar systems, respectively. This might be because many shuttle buses did not return immediately after the incidents were cleared, since they probably had to transport passengers on board to the shuttle route terminal point before they could go back to their original routes. However, a study that investigates the average speed, ridership and driver's behaviour using the actual bus operational (AVL) data is recommended to identify the causes of this increase in returning time. Indeed, understanding the reasons behind that would help in implementing actions to reduce the returning time. Similar patterns can be observed regarding the average recovery time per bus by weekdays vs. weekends, by time period, and by month, relative to the system response time, with longer returning times being observed. The higher efficiency observed for peak hours is also shown in the incident recovery time, highlighting the need to further improve recovery times for incidents on non-peak periods. In addition to the above cases, and for long delay incidents, with an average of 6 hours (about 70 incidents), a total of 256 buses went back directly to their routes before the clearance of the incident with a total average recovery time of 188 minutes. 
Figure 7: A- Average recovery time by mode, B- Average recovery time by weekday vs. weekend, C- Average recovery time by time period, and D- Average recovery time per bus over the months of year.

\section{INCIDENT ANALYSIS}

This section explores the most frequent incidents that occurred in Toronto's streetcar and subway networks in 2015, which is important to highlight how transit agencies react differently to different type of incidents. The analysis reports on the average incident delay, number of buses requested and assigned, and response time by incident type. Table 2-A indicates that over $30 \%$ of major streetcar incidents were caused by surface traffic accidents. However, these incidents were cleared rapidly, resulting in shorter delays (53.6 min per incident) and requiring fewer shuttle buses (1.8 buses per incident) than the average values for all major streetcar incidents ( 87 minutes and 2.3 buses, respectively). On the other hand, the overhead wire problem, resulting in a 2.5-hour delay (152 minutes) on average, led to the largest number of shuttle buses utilized. However, cold weather (including snow conditions) was to blame for the highest rate of delay for streetcars, with an average delay of more than 7 hours per incident. To give an example, on January $7^{\text {th }} 2015$, a large segment of streetcar Route 506 was down from 11:06 AM to 7:43 PM due to cold weather and snow on the ground. In response to that, the TTC requested 20 shuttle buses, 17 of which arrived and served on the temporary shuttle route. Such long service delays could undoubtedly have substantial impacts on the perception of passengers, both streetcar users travelling on the shuttle service and riders of bus routes from which shuttle buses were deployed. This is particularly important in the winter season, where passengers are more sensitive to any increase in their outdoor times including their waiting and walking times (Lam and Morrall 1982). The response time for all incident types ranged between 16.8 to 36.8 
401 minutes, except for cold weather incidents that suffered from a much longer response time of 40261.3 minutes. Regarding the subway system incidents, Table 2-B shows that power problems, 403 which are often related to cold weather and system level failures, led to the longest delay in the 404 subway system as well as the longest response time. Fire, smoke and burning odour was the most 405 frequent subway incident type, but each incident was cleared swiftly for most of the cases.

[TABLE 2: TO BE ADDED HERE]

\section{CONCLUSIONS AND RECOMMENDATIONS}

409

Transit agencies are constantly faced with the challenges of managing disrupted transit service, which often requires utilizing additional resources and diverting existing ones from one location to another in an effort to minimize user discomfort and delays. The primary aim of this study was to explore in more detail one of the common disruption management strategies, namely bus bridging, which transit agencies employ in response to major service interruptions in their urban rail systems. This strategy involves retracting buses from existing scheduled services to offer an emergency shuttle service to compensate for rail service interruptions. This strategy has been used widely by transit agencies, with no thorough analysis of its diverse aspects and impacts. Thus, a major contribution of this research is its examination of the different aspects and impacts of employing this strategy using the large-case multimodal transit system of Toronto as a case study, which provides a unique opportunity to understand the effects of not only subway service interruptions but also streetcar interruptions. In order to do that, the paper explored the magnitude and impacts of emergency shuttle service deployment, as well as the system response 
422 and recovery times using detailed subway and streetcar shuttle service reports collected in 2015

423 by the TTC. Such a dataset, which is usually hard to access, provided very detailed information

424 regarding the bus service retracting problem at the system level.

425 The paper shows considerable fluctuations in the number of incidents, number of

426 requested and assigned buses as well as the system's response and recovery times. These

427 fluctuations are not only within mode, but also across the two modes analyzed in this paper (i.e., 428 subway and streetcar), highlighting the challenge of managing disruption along the two systems.

429 Furthermore, this research highlights the need of more flexible protocols that recognize the

430 variations in system response and recovery times throughout the year, especially during the

431 winter season. In fact, additional categories, or different protocols, are needed to be added to

432 previous managerial emergency response frameworks proposed in the literature (Pender et al.

4332013 ) as well as to the one used by the TTC. These frameworks, which are based only on

434 disruption characteristics of duration, cause, time of day, and location, need to account for 435 seasonal changes in service response and recovery time as observed in the case of Toronto. In

436 addition, a study that develops new protocols for an integrated approach to retracting buses from

437 regular routes in combination with reserved spare buses (and drivers) according to the city

438 context is recommended. This would be important for some cities that experience special

439 seasonal weather conditions. For example, new protocols that facilitate faster response and

440 recovery times during the winter months should be developed, since passengers during this time

441 of year are more sensitive to any increase in their outdoor times including their waiting and

442 walking times (Lam and Morrall 1982).

One of the contributions of this study is developing a detailed framework to understand

444 the different aspects of the shuttle bus service workflow based on a case study of the large-case 
445 multimodal transit system of Toronto. More specifically, this study broke down the system 446 response time into three major components: Initial Response Time, Bus Pull out Time and Bus

447 Deadhead time. Similarly, it broke down the bus system recovery time into Bus Service Time on 448 the shuttle service and Bus Returning Time to the original routes. It also classified the shuttle 449 buses into three categories, including buses requested by the control centre, buses assigned by 450 divisions, and buses that actually arrived to the disruption location and provided the required 451 emergency service. Also, it developed the Division Response Rate measure to account for the 452 number of assigned vs. requested buses by division. This framework could be adapted for other 453 transit agencies to compare the relative performance across agencies, monitor trends over time 454 for individual agencies, and understand the impacts of new response management strategies. Another policy implication of this study is the need to employ a specific protocol to 456 initiate the emergency shuttle bus service by sourcing buses from scheduled service. An effective 457 communication plan is required in order to alert promptly the passengers of the affected bus 458 routes about the removal of scheduled trips. This is an important issue since, since passengers 459 tend to overestimate their waiting time compared to the actual wait time when it is imposed by 460 others (e.g., transit system) whereas they accurately estimate their waiting time when they 461 themselves choose to wait (Hess et al. 2004). Thus, by informing passengers, through social 462 media for example or by using transit apps alerts and bus stop variable message signs, about the 463 removal of some trips and the reasons behind that would help reduce the negative impact of 464 service cancellation on users' perception. Nevertheless, a more detailed study about the impact 465 of information provision and the type of used media on bus user travel behaviour and satisfaction 466 is recommended. 
While the challenge of sourcing the adequate number of buses from the existing

468

469

470

471

472 explored. In other words, fewer buses should be retracted from far locations, since these buses

473 would take more response and recovery times, leading to longer cancelled times for their users

474 than users of nearby routes. Thus, providing more flexibility in the percentage of required buses

475 from each division according to its location and incident location will mean a higher overall

476 system efficiency.

477

478

479

480

481

482

483 Furthermore, the number of retracted (or assigned) buses per route is not always proportional to

484 its ridership level and frequency, which highlights a considerable impact on the users of low

485 frequency bus routes. This study also shows considerable challenges in terms of longer recovery

486 and response times during weekends and off-peak periods, which can be attributed to the reduced

487 bus fleet in service during these periods, with less available trippers for retraction. This limits the

488 shuttle bus options for the transit agency and prolongs the system response and recovery time as

489 well as the overall decision making process. 
The previous observed challenges call for a better and more sophisticated tool for the

491

492

493

494

495

496

497

498

499

500

501

502

503

504

505

506

507

508

509

510

511

optimal design of the bus bridging strategy. In fact, the presented study provides useful and necessary insights which serve to inform ongoing work by the authors on developing such a tool.

The study also serves as a baseline against which to compare the new optimal bus bridging analytics. This optimal strategy could be formulated to minimize the system total user costs in terms of bus and subway users' total waiting and travel times. The solution of this optimization problem can be based on several inputs including: subway incidents location, start time and expected duration; subway through and local user volumes; and total number of required buses from a route and their response and recovery times (i.e., Pull out Time, Deadhead time and Returning time). The optimal strategy must respect some service quality constraints such as making sure that gaps in the bus service due to pulled buses are still within the headway policy of the transit agency, and all requested buses arrive and serve in the shuttle service, which would help in providing more efficient and comprehensive bus bridging solutions.

\section{ACKNOWLEDGEMENTS}

The authors gratefully acknowledge Erin Wemyss and Kenny Ling from the Toronto Transit Commission (TTC) for providing the data used in the paper and for their feedback and comments that helped improve the study. The authors would like to acknowledge the members of Nexus research group, particularly Rachel Ye and Dennis Wu for helping in the data entry. This research was funded by Natural Sciences and Engineering Research Council of Canada (NSERC) and the Ontario Research Fund (ORF). The ideas and findings presented in this paper represent the authors' views in an academic exercise.

\section{REFERENCES}


512 American Public Transportation Association (APTA). 2013. APTA transit ridership report,

513 Second Quarter, 2013. American Public Transportation Association (APTA), Washington, D.C.

514 Bates, J., Polak, J., Jones, P., and Cook, A. 2001. The valuation of reliability for personal travel.

515 Transportation Research Part E: Logistics and Transportation Review 37(2-3): 191-229.

516 Codina, E., and Marin, A. 2010. A design model for the bus bridging problem, Lyons, France.

517 Darmanin, T., Lim, C., and Gan, H. 2010. Public railway disruption recovery planning: A New

518 recovery strategy for Metro Train Melbourne, Melaka, 7-10 December 2010

519 Diab, E., and El-Geneidy, A. 2013. Variation in bus transit service: Understanding the impacts of 520 various improvement strategies on transit service reliability. Public Transport 4(3): 209-231.

521 Diab, E., and Shalaby, A. 2017. Understanding the impacts of subway's system interruptions on 522 streetcar and bus transit service performance, Santiago, Chile.

523 Diab, E., van Lierop, D., and El-Geneidy, A. 2017. Recommending transit: Disentangling users’ 524 willingness to recommend transit and their intended continued use. Travel Behaviour and 525 Society 6: 1-9.

526 Hess, D., Brown, J., and Shoup, D. 2004. Waiting for the Bus. Journal of Public Transportation 527 7(4): 67-84.

528 Kepaptsoglou, K., and Karlaftis, M. 2009. The bus bridging problem in metro operations: 529 conceptual framework, models and algorithms. Public Transport 1(4): 275-297.

530 Lam, W., and Morrall, J. 1982. Bus passenger walking distances and waiting times: A summer531 winter comparison. Transportation Quarterly 36(3): 407-421. 
532 Lin, T. 2017. Transit user mode choice behaviour in response to TTC rapid transit service 533 disruption Department of Civil Engineering, University of Toronto, Toronto.

534 Lin, T., Shalaby, A., and Miller, E. 2017. Transit user behaviour in response to subway service 535 disruption, London, Ontario, Canada.

536 Nam, D., Park, D., and Khamkongkhun, A. 2005. Estimation of value of travel time reliability. 537 Journal of Advanced Transportation 39(1): 39-61.

538 Noland, R., and Polak, J. 2002. Travel time variability: A review of theoretical and empirical 539 issues. Transport Reviews 22(1): 39-54.

540 Ontario Ministry of Finance. 2015. Ontario population projections update, 2015-2041. Available 541 from http://www.fin.gov.on.ca/en/economy/demographics/projections/ [accessed 24 July 2016 542 2016].

543 Pender, B., Currie, G., Delbosc, A., and Shiwakoti, N. 2013. Disruption recovery in passenger 544 railways: International survey. Transportation Research Record: Journal of the Transportation 545 Research Board(2353): 22-32.

546 Pender, B., Currie, G., Shiwakoti, N., and Delbosc, A. 2015. Economic viability of bus bridging 547 reserves for fast response to unplanned passenger rail disruption. Transportation Research 548 Record: Journal of the Transportation Research Board(2537): 13-22.

549 Schmöcker, J., Cooper, S., and Adeney, W. 2005. Metro service delay recovery: Comparison of 550 strategies and constraints across systems. Transportation Research Record: Journal of the 551 Transportation Research Board 1930: 30-37. 
552 TTC. 2012. Surface ridership 2012. Available from

553 https://www.ttc.ca/About the TTC/Transit_Planning/Surface_Ridership_2012.jsp [accessed 24

554 July 2016].

555 TTC. 2013. TTC Operating Statistics 2013. Available from

556 https://www.ttc.ca/About the TTC/Operating_Statistics/2013.jsp [accessed May 2nd 2016].

557 van der Hurk, E., Koutsopoulos, H., Wilson, N., Kroon, L., and Maróti, G. 2016. Shuttle

558 planning for link closures in urban public transport networks. Transportation Science 0(0): null.

559 Wang, Y., Guo, J., Currie, G., Dong, W., and Pender, B. 2014. Bus bridging disruption in rail

560 services with frustrated and impatient passengers. IEEE Transactions on Intelligent

561 Transportation Systems 15(5): 2014-2023. 
Table 1: Number of required buses to be retracted for the shuttle service

\begin{tabular}{|c|c|c|c|c|c|c|c|}
\hline \multirow{2}{*}{\multicolumn{2}{|c|}{$\begin{array}{l}\text { Expected incident time } \\
\text { Closed subway stations }\end{array}$}} & \multicolumn{3}{|c|}{+30 MINS } & \multicolumn{3}{|c|}{1 - 30 MINS } \\
\hline & & $1-4$ & $5-9$ & $10+$ & $1-4$ & $5-9$ & $10+$ \\
\hline \multicolumn{2}{|c|}{ Percentage $(\%)$} & $3.33 \%$ & $6.66 \%$ & $10 \%$ & $1.67 \%$ & $3.33 \%$ & $5.00 \%$ \\
\hline Time period & $\begin{array}{l}\text { Number of buses } \\
\text { for regular service }\end{array}$ & \multicolumn{6}{|c|}{ Number of required buses for the shuttle service } \\
\hline $6: 00-9: 00$ & 1325 & 44 & 88 & 133 & 22 & 44 & 66 \\
\hline 9:00-15:00 & 881 & 29 & 59 & 88 & 15 & 29 & 44 \\
\hline $15: 00-19: 00$ & 1426 & 47 & 95 & 143 & 24 & 47 & 71 \\
\hline 19:00-22:00 & 819 & 27 & 55 & 82 & 14 & 27 & 41 \\
\hline $22: 00-1: 00$ & 506 & 17 & 34 & 51 & 8 & 17 & 25 \\
\hline
\end{tabular}

*Source: TTC's Route Management Department 
Table 2: Five most frequent reasons for streetcar and subway incidents

\begin{tabular}{lccccc}
\hline Reason & Incidents & $\begin{array}{c}\text { Buses } \\
\text { Requested }\end{array}$ & $\begin{array}{c}\text { Buses } \\
\text { Assigned }\end{array}$ & $\begin{array}{c}\text { Average } \\
\text { Response Time } \\
\text { (min) }\end{array}$ & $\begin{array}{c}\text { Average Clear } \\
\text { Time (min) }\end{array}$ \\
\hline & \multicolumn{7}{c}{ A. Streetcar incidents } \\
\hline Auto/Pedestrian Accident & 372 & 1.9 & 1.8 & 21.7 & 53.6 \\
Disabled Streetcar & 145 & 1.9 & 1.7 & 18.0 & 52.9 \\
Cold Weather & 83 & 2.6 & 2.3 & 61.3 & 453.6 \\
Overhead Wire Down & 30 & 3 & 2.4 & 31.6 & 152.8 \\
Working Fire/Fire on Streetcars & 15 & 2.2 & 2 & 36.8 & 85.2 \\
Medical Emergency & 11 & 1.9 & 1.7 & 16.8 & 21.8 \\
\hline Fire, Smoke or Burning Odour & 32 & B. Subway incidents & 30.8 \\
Power Problem & 14 & 12.5 & 6.2 & 31.5 & 307.2 \\
Suicides on the Subway Tracks & 12 & 9.6 & 9.9 & 52.5 & 51.8 \\
Unauthorized at Track Level & 10 & 6.3 & 5.6 & 23.3 & 111 \\
Medical Emergency & 10 & 6.7 & 7.5 & 44.6 & 41.6 \\
Cold Weather & 7 & 5 & 4.7 & 20.8 & \\
\hline
\end{tabular}




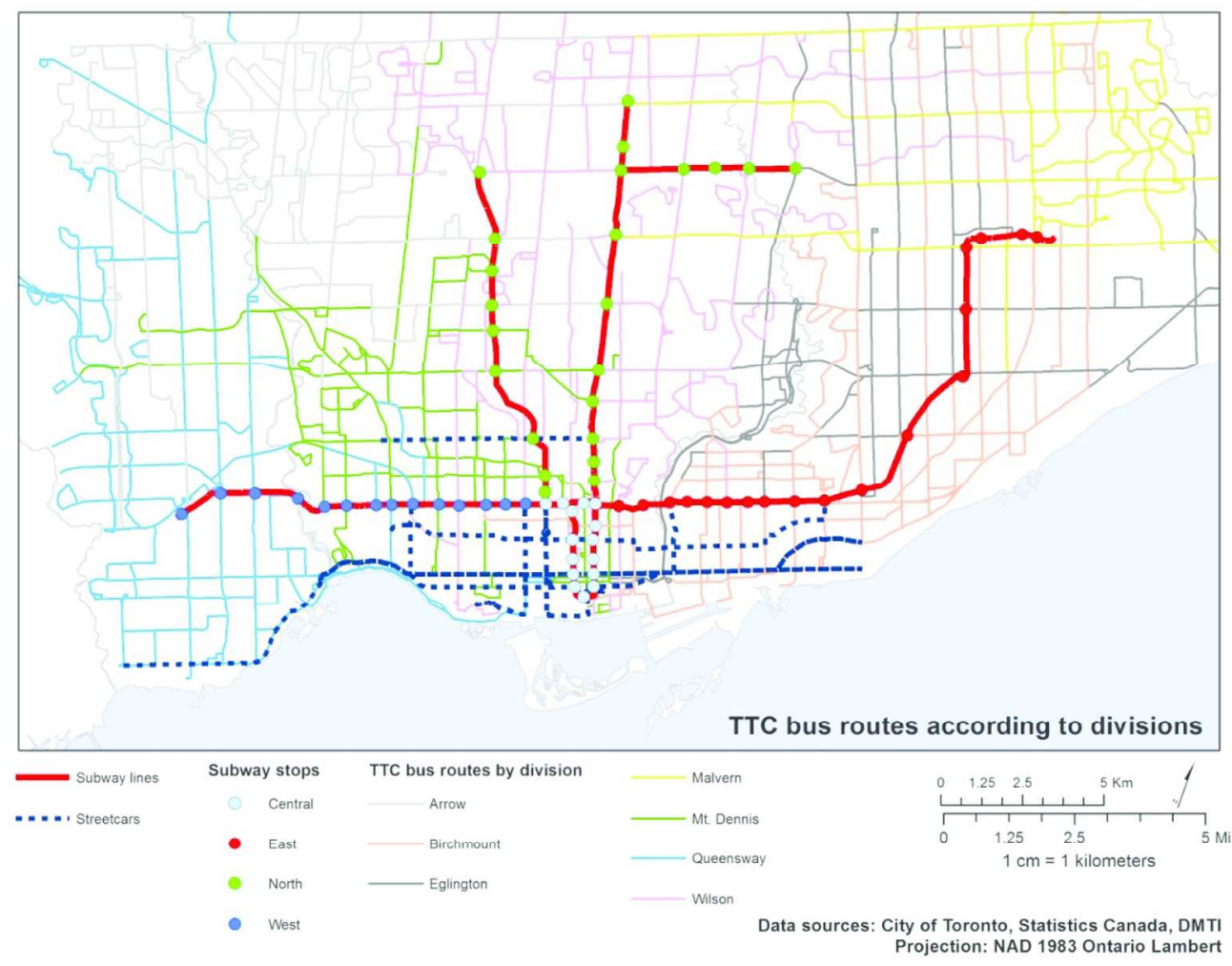

Figure 1: TTC system map of streetcar, subway and bus lines $146 \times 112 \mathrm{~mm}(300 \times 300 \mathrm{DPI})$ 


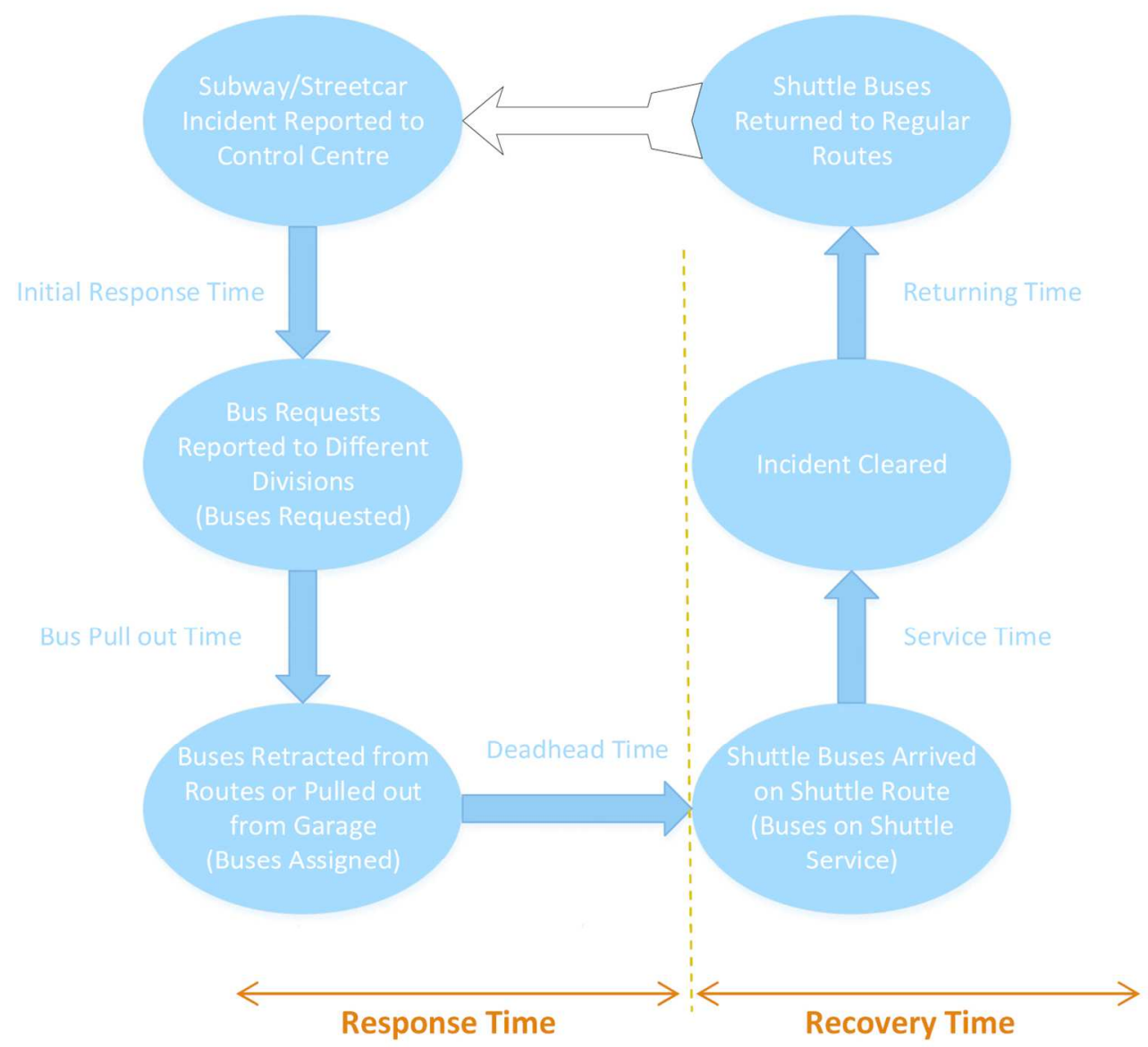

Figure 2: Regular workflow cycle for a shuttle bus service.

$131 \times 129 \mathrm{~mm}(300 \times 300 \mathrm{DPI})$ 


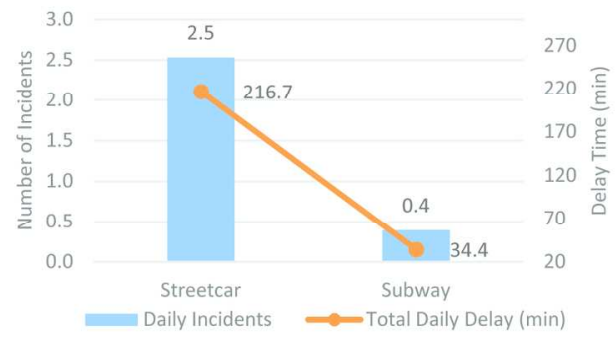

A

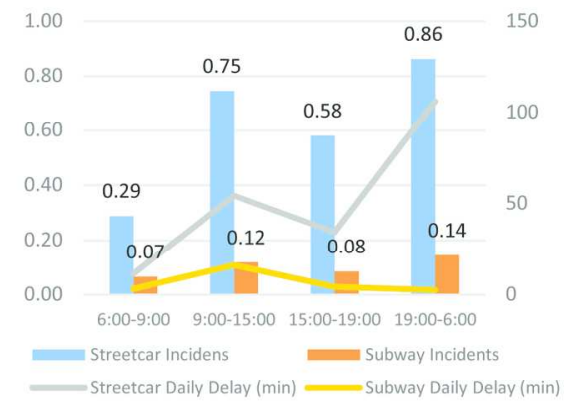

C

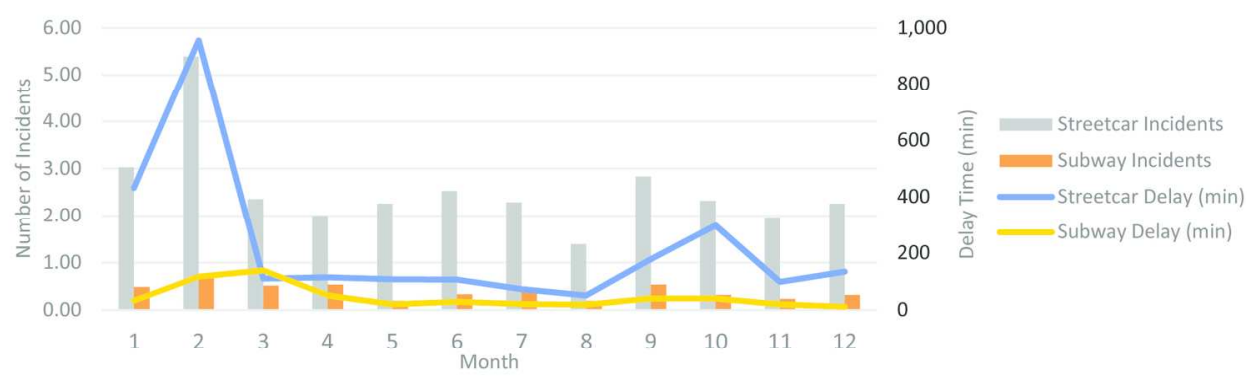

E

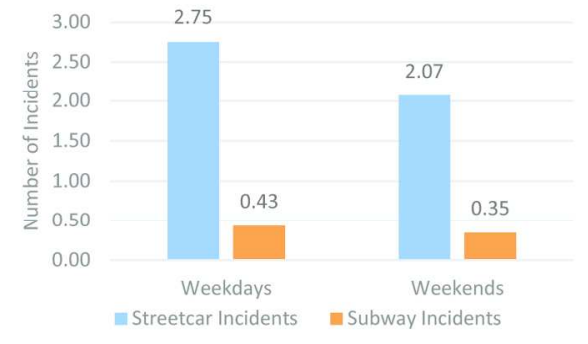

B

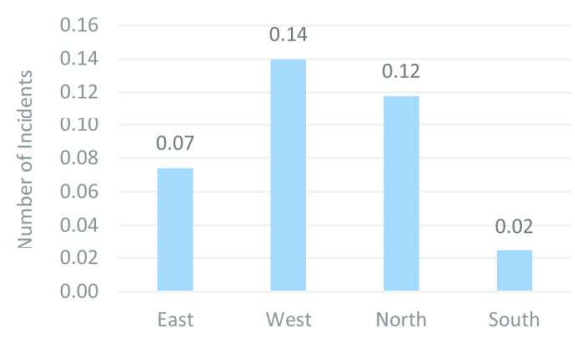

D

Figure 3: A- Daily incidents and total daily delay by mode, B- Daily subway incidents by weekday vs weekend, C- Daily incidents and average delay by time period, D- Daily subway incidents by location, and EDaily incidents and total daily delay by month.

$168 \times 199 \mathrm{~mm}(300 \times 300 \mathrm{DPI})$ 


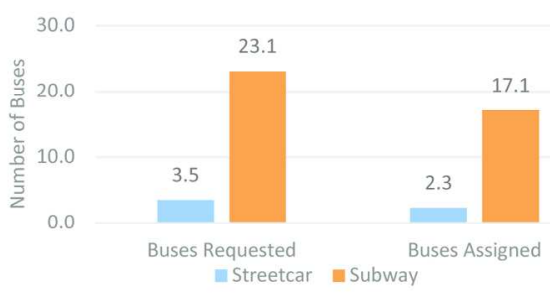

A

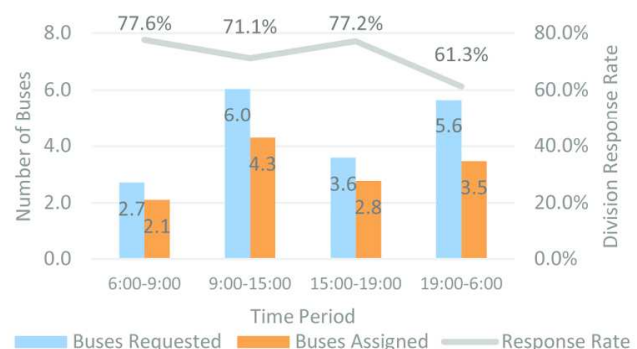

C

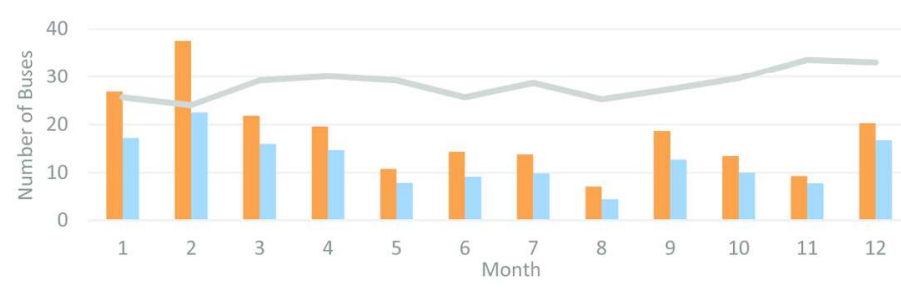

F

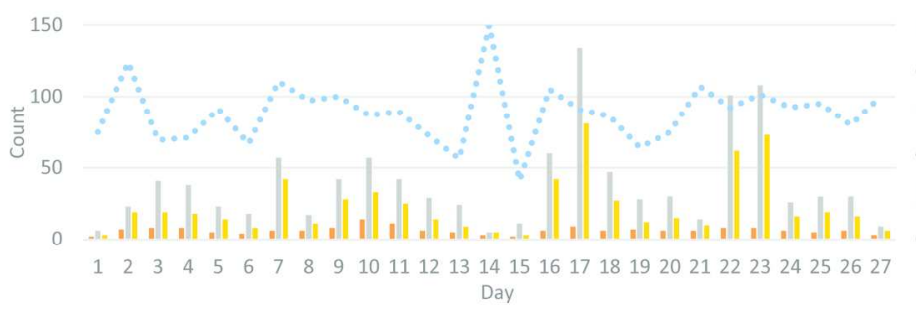

F

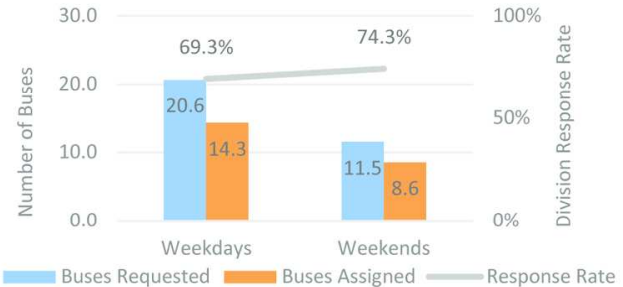

B

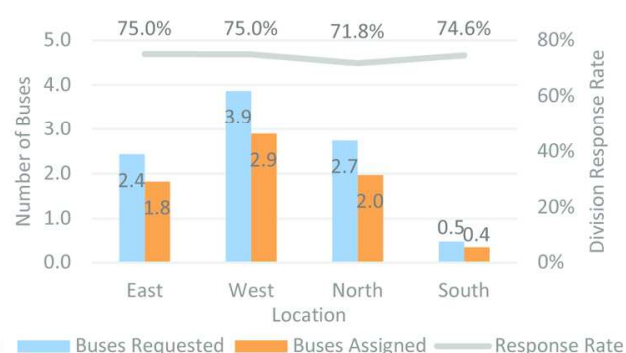

D

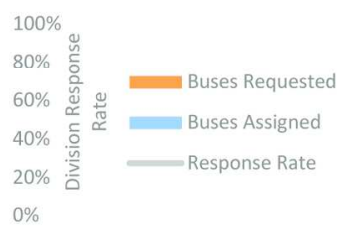

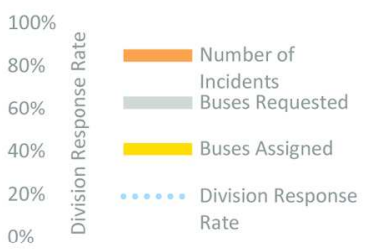

Figure 4: A- Buses requested and assigned per incident by mode, B- Daily buses requested and assigned by weekday vs. weekend, C- Daily buses requested and assigned by time period, D- Daily buses requested and assigned by location (for the subway system), E- Daily buses requested and assigned by month, and F-. Daily shuttle bus service analysis for February 2015.

$169 \times 202 \mathrm{~mm}(300 \times 300 \mathrm{DPI})$ 


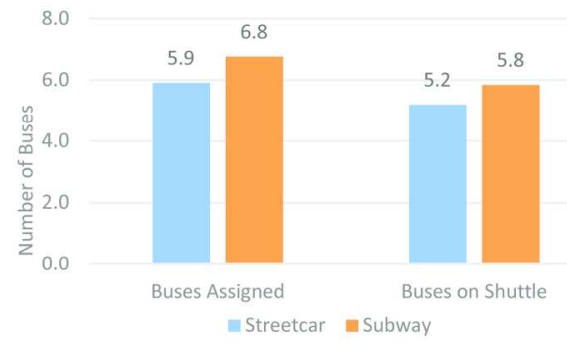

A

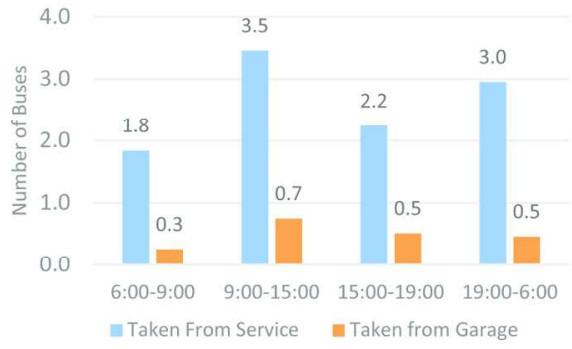

B
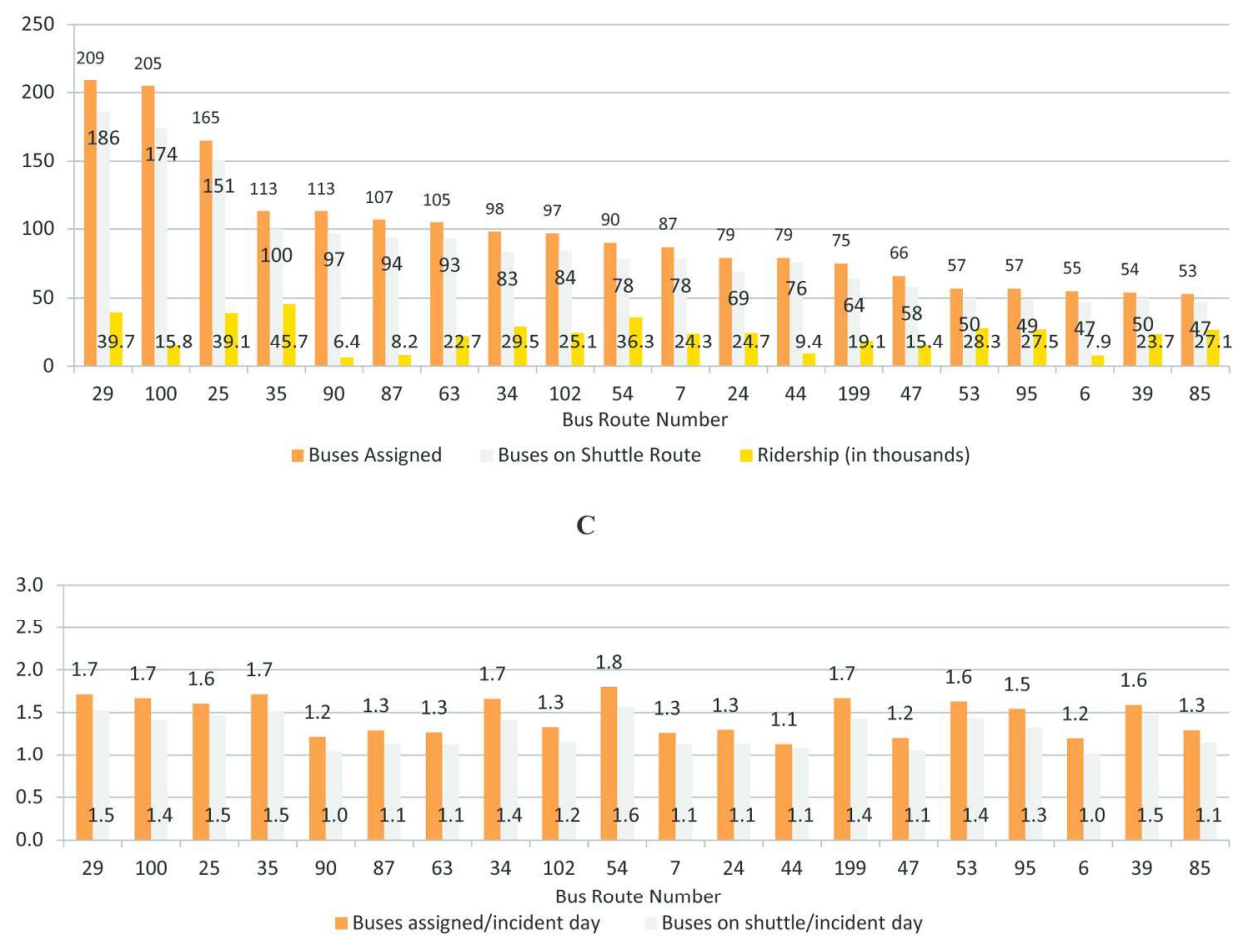

D

Figure 5: A- Daily assigned and in-shuttle buses by mode, B- Daily buses retracted from scheduled service and garages by time period, C- Top 20 bus routes that supplied buses for shuttle service in 2015, and DBuses retracted from scheduled service on the incident days.

$169 \times 196 \mathrm{~mm}(300 \times 300 \mathrm{DPI})$ 


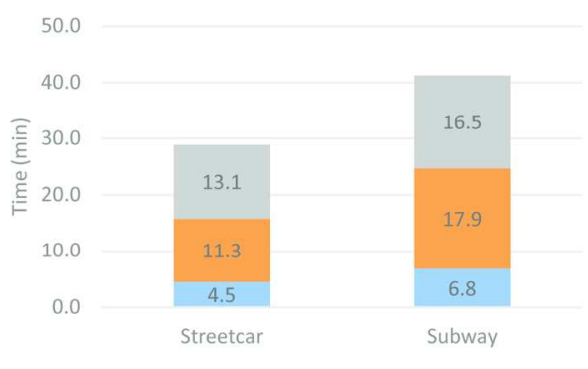

- Initial Response Time $=$ Bus Pull Out Time $=$ Deadhead Time

A

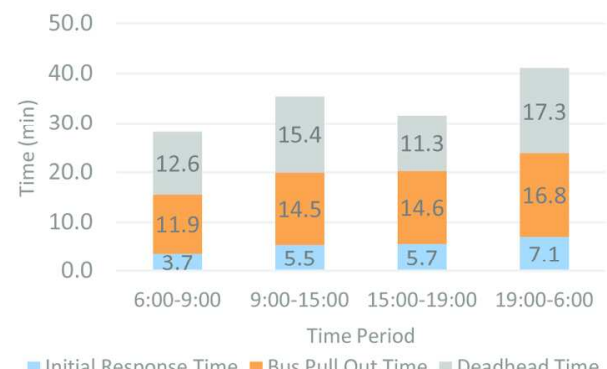

C

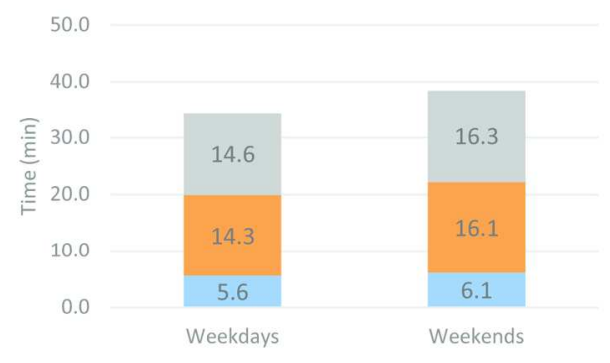

= Initial Response Time $\mathbf{m}$ Bus Pull Out Time $\equiv$ Deadhead Time

B

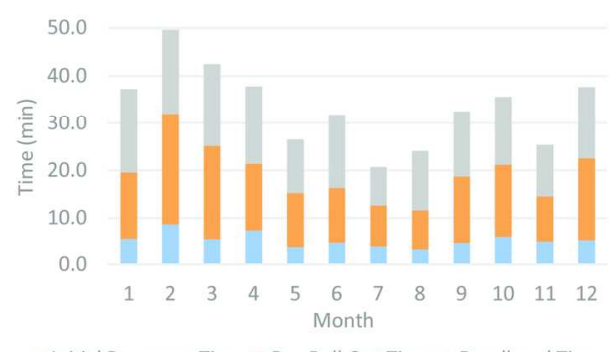

m Initial Response Time $\approx$ Bus Pull Out Time $m$ Deadhead Time

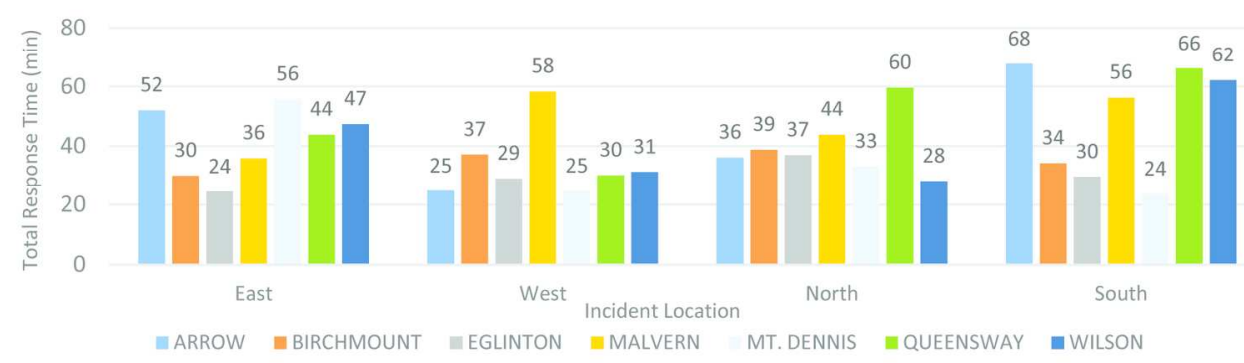

$\mathbf{E}$

Figure 6: A- Average bus response time by mode, B- Average bus response time by weekday vs. weekend, C- Average bus response time by time period, D- Average bus response time by months, and E. Average bus response time by location in subway network and division.

$164 \times 187 \mathrm{~mm}(300 \times 300 \mathrm{DPI})$ 


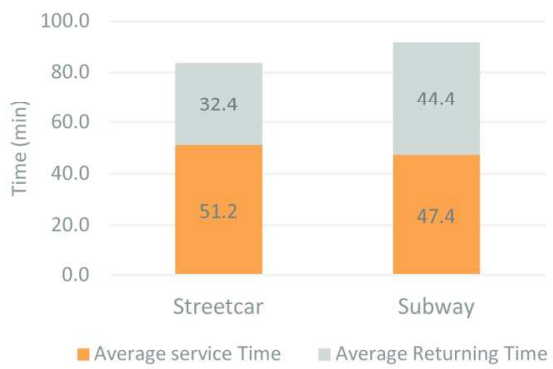

A

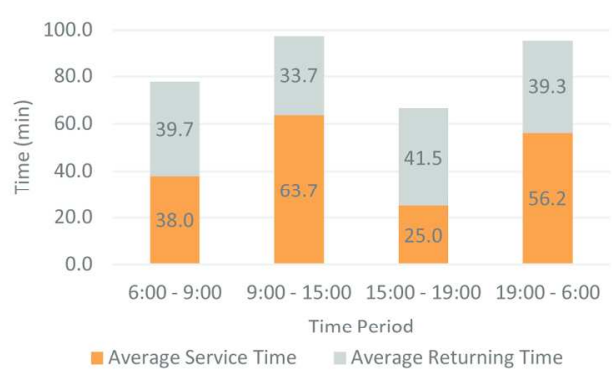

C

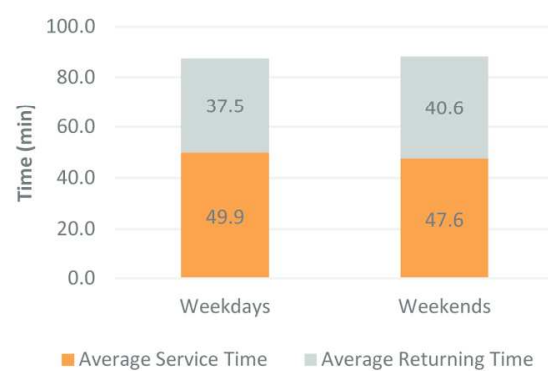

B

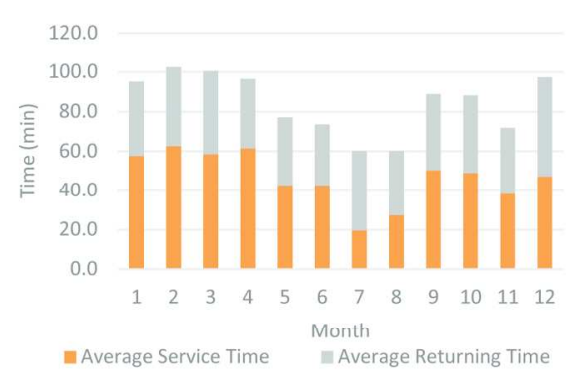

D

Figure 7: A- Average recovery time by mode, B- Average recovery time by weekday vs. weekend, CAverage recovery time by time period, and D- Average recovery time per bus over the months of year.

$169 \times 126 \mathrm{~mm}(300 \times 300 \mathrm{DPI})$ 